\title{
Development of Dynamic Programming Algorithm for Maintenance Scheduling Problem: A Conceptual Paper
}

\author{
Z. A. M. Fauzi and * M. A. Mansor \\ Faculty of Engineering Technology, University Malaysia Pahang, 26300, Kuantan, Pahang, Malaysia \\ "Email: ariffin@ump.edu.my
}

\begin{abstract}
Maintenance is one of the important methods that can be used to ensure the machines and equipment can operate within the best condition. A good scheduling for maintenance will maintain the proper performance since all resources will be utilised effectively as the underused of the resource can be reduced. The schedule created ensures the particular task was done within the time provided. What, when, where and how the certain operation will be done will be stated to make sure the planned activity going smoothly without any delays. However, the uncertainty that happened during the maintenance needs to be taken into account to create the fully optimised schedule. This change will affect the schedule developed and caused delays to the scheduled task. Once the beginning task is delayed, the following work will also be postponed. Thus, a mathematical model for maintenance scheduling for repairable and inspection of the machines and equipment was proposed to deal with the uncertainty that happened. This dynamic model will create the new schedule for the maintenance scheduling and help the manager in the decision-making process on where to assign the maintenance team when the uncertainty happened.
\end{abstract}

Indexed Terms- maintenance, scheduling, maintenance scheduling, dynamic programming

\section{INTRODUCTION}

Scheduling is the arrangement of something to happen at a particular time. Baker [1] states that scheduling is the allocation of the machine over time to perform a task. Schedule describes guidance and plans to run or to operate an operation. Thus, a proper scheduling will maintain the good performance since all resources will be used effectively as the underused of the resource can be reduced. As for industries that are implementing a proper scheduling, cost such as operational cost [2] and labour cost can be reduced when the operation time is fully optimized. Any job delay also can be prevented. For example, in a factory, the manager needs to create a schedule for the operation of the plant from the labour, maintenance and other operation so that the production or operation will not be delayed.

Maintenance is the act of keeping something in good condition by checking or repairing it regularly. Maintenance ensuring the equipment or machines works as new. Maintenance can be planned and unplanned [3]. Moreover, maintenance planning is also a better way to increase not only the productivity but also some performance indicators such as overall equipment effectiveness [4]. Thus, scheduling is one of the methods that can be used to plan and schedule the maintenance process that needs to be done. The maintenance schedule is crucial to eliminate unexpected replacement and break down, and it also can keep costs down because the maintenance is done as the problems detected before an expensive repair is needed. A proper schedule is the efficient and economical ways to do the maintenance. 
The schedule created ensures the particular task was done within the time provided. In order to get the optimise maintenance schedule, any unexpected circumstances during maintenance activities should be taken into consideration. Delays will happen to the scheduled task due to this uncertainty. This will affect the schedule developed because the following schedule will be affected since the beginning task is delayed. Thus, a mathematical model that can deal with the unexpected circumstance or uncertainty that happened during the maintenance activities will be proposed. This method used to solve a complex problem by breaking it down into a collection of simpler sub problems, solving each of those sub problems just once, and storing their solutions and it is usually used for optimisation problem such as the shortest path, optimum decisions for investment, etc. This model will create the new schedule that will help the manager in the decision-making process when the uncertainty happened so that the manager will know where to assign the maintenance team.

\section{LITERATURE REVIEW}

Maintenance is a necessary action for retaining or restoring or maintaining a piece of equipment, machine, or system to the specified operable condition to achieve its maximum useful life. According to M.A. Mansor [5], maintenance efficiency and effectiveness play a significant role in an organisation's success and survival, and it also ensures that the equipment is functioning at its optimal level. There are two categories of maintenance which is planned and unplanned as shown in Figure 1. Figure 1 indicates that preventive maintenance and corrective maintenance are classified as planned maintenance. There are two categories of preventive maintenance; fixed and predictive. Fixed maintenance is said to be costly because it requires periodic maintenance before the breakdown occurs.

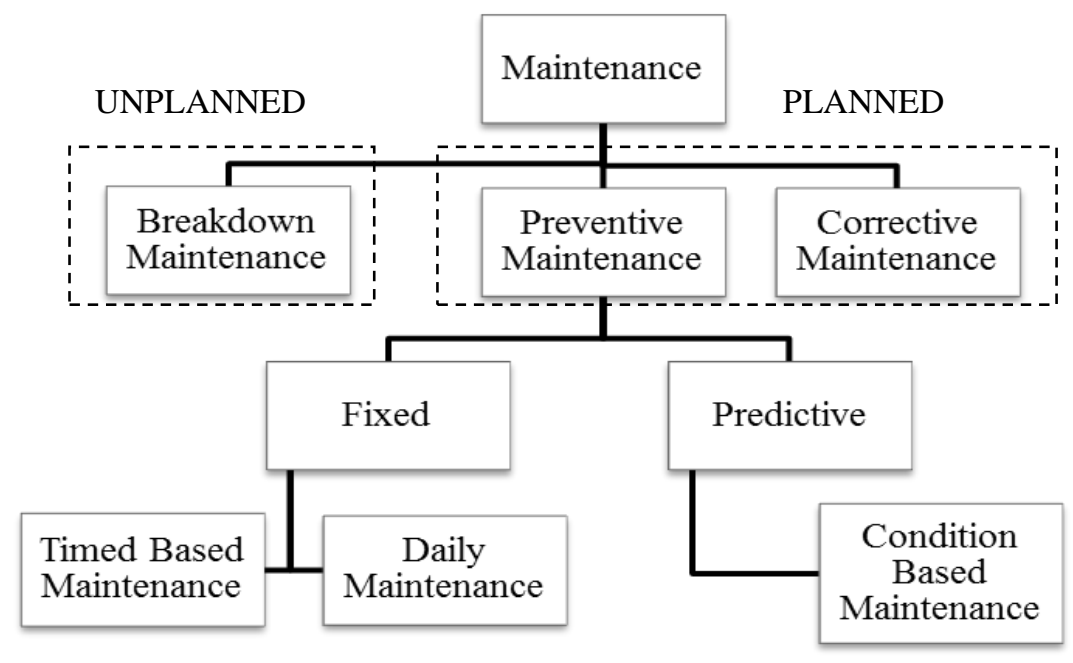

Figure 1: Type of Maintenance [5]

There are two types of fixed preventive maintenance: time-based maintenance and daily maintenance. For predictive preventive maintenance, there is only one type which is condition based maintenance. Predictive maintenance is designed to predict when maintenance should be done by determining the state of the in-service equipment. Condition based maintenance is one type of the predictive maintenance where Condition Diagnosis Technology is used to observe the status of machines and equipment and then predict its lifespan. Meanwhile, breakdown maintenance is classified as unplanned maintenance because the repair or exchange of parts was done after a sudden breakdown or malfunction of the equipment and this type of maintenance strategy used to be typical for many years [6].

Froger et al., [7], Ye, et al., [8] and Azhar and Mansor [9] said that there are two types of maintenance which are preventive maintenance and corrective maintenance. Preventive maintenance is a process which ensures the machine or equipment always in the best condition by performing daily routine inspection or also known as daily maintenance. Meanwhile, corrective maintenance is the maintenance task that is conducted to improve the bad spots of machines and equipment and reduce 
breakdown [3].

The maintenance schedule is then being considered in a company where different maintenance situation exist [10]. Six elements need to be bringing together for scheduling which are mechanics, tools, material or parts, availability of unit to be serviced, information need to complete the job and necessary permission. Scheduling is the process of adding start and finish information to the task order dictated by the sequence where the sequence is the process of defining the order in which the set of jobs to be completed are done [11]. Reklaitis [12] said that planning and scheduling of production facilities is a decision-making process that determines what, when, where, and how $(3 \mathrm{~W} 1 \mathrm{H})$ to produce a set of products.

- What refers to the amount of product made or the jobs need to be done at that particular time.

- Where refers to the specific site of the particular operation.

- When refers to the timing of the specific operation.

- How relates to how the job will be done, the equipment used, manpower needed to handle the job, etc.

\section{APPLICATIONS OF DYNAMIC PROGRAMMING}

Dynamic programming is a useful mathematical method for making a sequence of the interrelated decision. Figure 2 explains that the dynamic programming technique starts with a small subset of the original problem, i.e. a sub problem and find the optimal solution for the sub problem. Then, the sub problem was enlarged to find the current optimal solution from the sub problem until the entire problem is solved. Terminologically in dynamic programming, a sub problem is called a stage. Thus, the primary problem can be decomposed into several stages and then solved stage by stage.

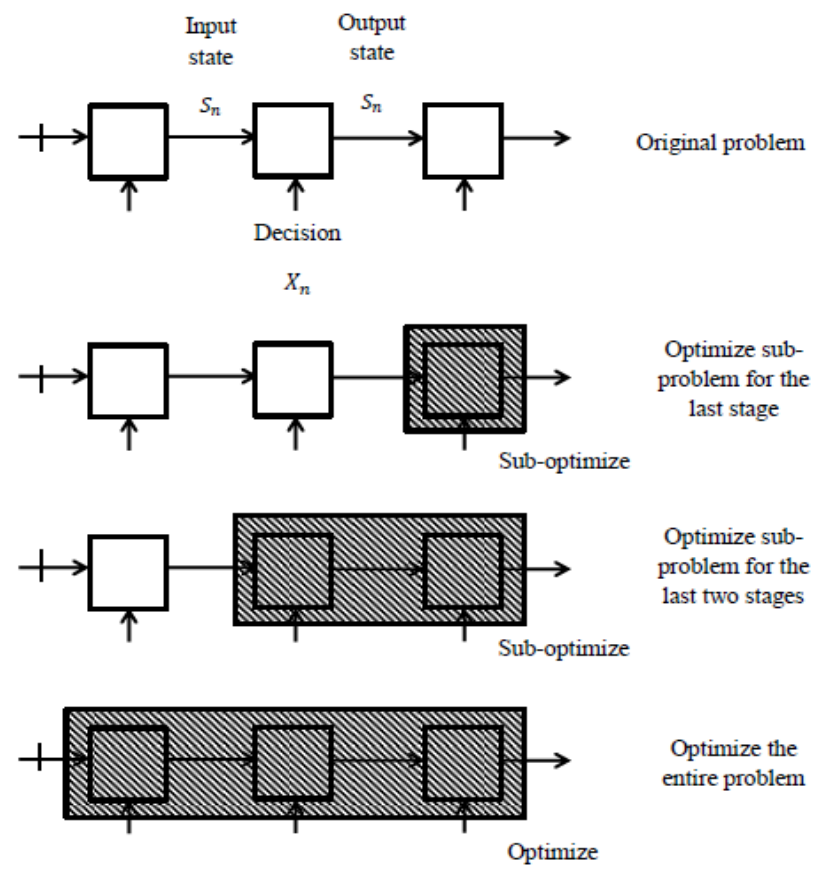

Figure 2: Dynamic Programming for Optimization of Serial Systems [13]

There are two types of dynamic programming; deterministic and probabilistic. The focus will be on deterministic dynamic programming. The basic structure of deterministic dynamic programming is shown in Figure 3. The deterministic dynamic programming is where the state of the next stage is entirely determined by the state and policy decision of the current stage. 


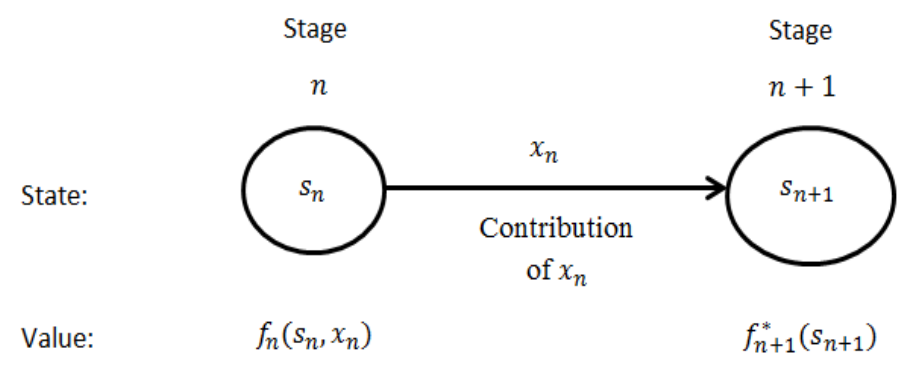

Figure 3: The Basic Structure of Deterministic Dynamic Programming [14]

Figure 3 shows the diagram of the basic structure of deterministic dynamic programming. At stage $n$, the state will be $s_{n}$ Making policy decision, $x_{n}$ then the process will move to state $s_{n+1}$ and stage $n+1$. Then, the objective function will be $f_{n+1}^{*}\left(s_{n+1}\right)$. The policy decision $x_{n}$ also makes some contribution where it combined these two quantities in an appropriate way provided $f_{n}\left(s_{n}, x_{n}\right)$. Optimization with the respect to $x_{n}$ gives $f_{n}^{*} s_{n}=f_{n}\left(s_{n}, x_{n}^{*}\right)$. After $x_{n}^{*}$ and $f_{n}^{*}\left(s_{n}\right)$ are found for each possible value of $s_{n}$, the solution procedure is ready to move back one stage.

\section{DEVELOPMENT OF DYNAMIC PROGRAMMING ALGORITHM FOR MAINTENANCE SCHEDULING}

The development of the dynamic programming starts with featuring the problems into the topology graph so that the problem can be understood clearly. The topology graph is a graphical illustration on how to model the dynamic programming for maintenance scheduling problem. Figure 4 shows the topology graph for the maintenance activity or maintenance places that might be chosen for maintenance process. There are more than one places need to be maintained daily, monthly or yearly, and the assigned location can be changed according to the uncertainty that happened. This topology graph is an example of the flexibility of the schedule created according to the situation happened.

Based on Figure 4, the $m_{1}$ is the state where the first maintenance activity started. The $m_{1}$ state can be referred to the maintenance activity in a station or relates to the maintenance places if the company has several stations in different locations that need to be maintained. The maintenance schedule starts with stage 1 which is the starting point of the maintenance process. For stage two, there are several places can be possibly chosen. These can be changed according to the situation. It can be more or less than the example in Figure 4. Then the process will continue until stage $n$ and stage $n+1$ followed the problems that are going to solve.

Figure 5 shows the simple network for the maintenance order, but in real life, the system will be more complex. The network shows the four stages maintenance schedule that starts from the stage 1 until stage 4. Each stage consists a lot of stations that need to be maintained within one day, and time will be the biggest factor to reconsider the order of the maintenance schedule so that the fully optimised maintenance schedule can be created. The station is assumed to be in the different region within a state, and each station has its machines and equipment that will need to be maintained. 


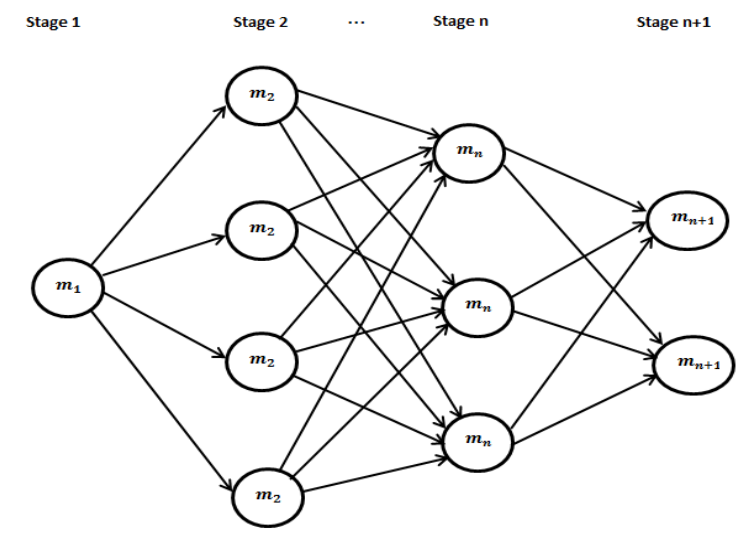

Figure 4: The Topology Graph

There are a lot of factors need to be considered when developing a schedule. The factors that will be significant are as follows:

- The distance from one station to the next station which will affect the time taken to arrive at the next station in the schedule

- Time taken for the maintenance at each station

- Number of component or machines that need to be inspected or maintain at each station which affects the time taken for the maintenance

- Number of manpower involved in each station

The uncertainty that happened during maintenance schedule will be taken into account as it will affect the schedule that was created. The following are the uncertainty that might affect the schedule developed.

- Availability of the transportation

- Availability of manpower

- Availability of compartment that will be replaced or maintained

- Unexpected ad hoc maintenance that needs to be maintained instantly

- Sudden failure or shut down of the station

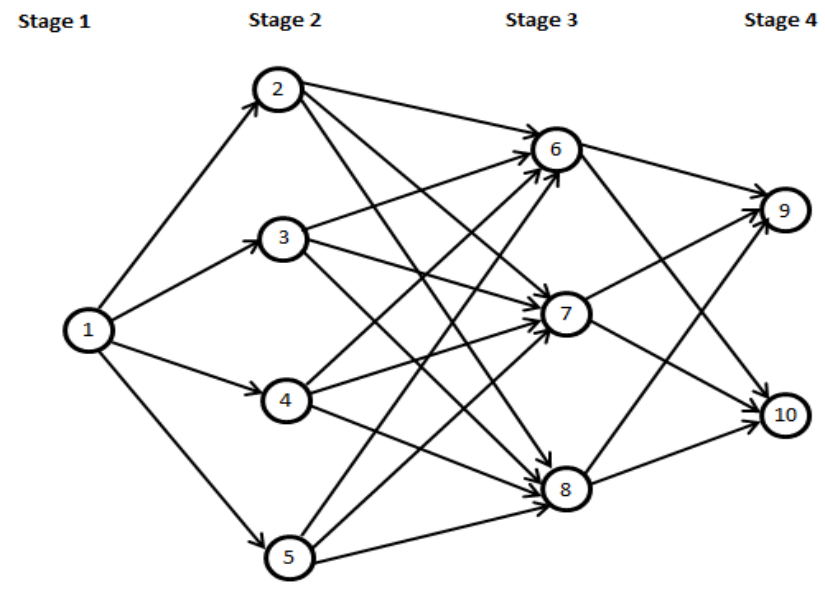

Figure 5: The Simple Network for Maintenance Scheduling Order 
From the Figure 5, each stage represents the maintenance scheduling sequence for a day. For stage 1 , the number 1 indicates the station number that needs to be maintained. The first station would be the most important to decide because the planning of it will affect the following stage schedule if delays happened on the previous stage. During the maintenance process at the station 1, the distance between the region will be the issues as the time from a station to a station will also affecting the schedule. Time taken for each activity at the station will be significant, and the number of the manpower involved also necessary because as the number of workforce increase, the time taken for each process will be less. The time consuming for the stage will be shorter. The number of component or machines that need to be maintained will also be the significant factor that affects the schedule.

For stage 2, there are four stations were considered to be maintained which is station 2, station 3 , station 4 and station 5 but only one can be maintained at the particular time. Four stations were considered to be maintained according to the possibilities and uncertainties that will happen. So, the next schedule can be station 2, station 3, station 4 or station 5 . The state of each stage can be varied according to the situation that happened during the maintenance processes. For example, the original schedule that was developed assigning the station 3 for the second stage of the schedule but suddenly ad hoc maintenance is needed at the other station. The other reasons that also will affect the maintenance schedule that was developed are the availability of the transportation and the availability of manpower. If there is no transport and manpower to do the maintenance, the job cannot be done and will not finish on time as these uncertainties causing postpone for maintenance job that needs to be done.

Moreover, the availability of compartment that needs to be replaced or maintained is one of the uncertainties that will happen. If the compartment is not ready yet, manpower needs to order, and for each order, there will be waiting period for receiving the order. During this period, maintenance activity cannot proceed, and delays will happen. The unexpected failure or shutdown of the station also needs to be considered. The matter of priorities is the most important at this stage. The decisionmaking process on where to assign the team for maintenance schedule will be a problem in stage 2 and the following stage. These uncertainties need to be considered so that the maintenance activities can be completed on time. The uncertainty that happens is the reason why the stage two has more than one station that needs to be chosen.

By considering the uncertainty that will happen during the maintenance process at stage 2 , the other parameters still will be calculated to make the decision which station will be maintained in stage 2. The parameter such as the distance from one station to the next station that will affect the time taken to arrive at the next station in the schedule, time taken for the maintenance of each station, number of component or machines that need to be inspect or maintain at each station which affect the time taken for the maintenance and number of manpower involved in each station will be calculate and the station that will be maintained will be chosen and included in the schedule. Both parameters and uncertainties will be significant to create a maintenance schedule for this research.

For stage 3 and stage 4, the process will be the same as the stage two where the uncertainty is sufficient to the maintenance scheduling order. The maintenance scheduling order will be flexible and can change according to the situation and uncertainty that happen. This type of schedule can help to avoid delays and the action taken will be faster. A dynamic programming model is proposed because dynamic programming is flexible as it can deal with the uncertainty that happened during the maintenance processed. The algorithm developed for maintenance scheduling problem was established based on the dynamic programming principle as shown in Figure 3. The following is the algorithm developed:

Stage $(n)$ : The state variables represent the state of the systems as it moves through the maintenance process. The stage is divided by each process. Therefore, the index $n$ represent given stage for each process, $n=1,2, \ldots, n, n+1$.

State $\left(m_{n k}\right)$ : The maintenance station in each stage. Therefore, the state variable $m_{n k}$ is set to be the maintenance station at stage $n$ where $k$ is the number of places that need to be maintained at each stage, $k=1,2,3, \ldots, k$.

$m_{n}=\left[m_{n 1}, m_{n 2}, \ldots, m_{n k}\right]$ 
Decision Variables $\left(x_{n k}\right)$ : The decision-making problem is where to assign the maintenance team or what maintenance needs to be done on the next stage with the purpose of optimising the time for each stage.

$x_{n}=\left[x_{n 1}, x_{n 2}, \ldots, x_{n k}\right]$

For decision variables, the parameters that are included in the algorithm are introduced. These parameters will be used to calculate to decide on where to assign the maintenance team. The model from Lieberman and Hillier [14] is implemented to state the overall problem mathematically. Let $T_{n i}$ be the time measure for allocating maintenance team to station $k$. Thus, the objective is to choose $x_{n 1}, x_{n 2}, x_{n 3}, \ldots, x_{n k}$ as to

$\min \sum_{i=1}^{k} T_{n i}$

Parameters:

d : Distance

$T \quad:$ Time

$t_{m k} \quad:$ Time taken for each maintenance activity at a station

$t_{d} \quad:$ Time taken for distance from current stage to next stage

$t_{N} \quad$ : Time taken for the total number of inspection during the maintenance process at stage $n$

$t_{\text {start }} \quad:$ Start time for maintenance process

$t_{\text {finish }}:$ Finished time for maintenance process

$N_{c} \quad$ : Number of component or machines that need to be inspected or maintain for the stage $n$ at state $k$.

$P_{\text {num }} \quad$ : Number of manpower involved in each plant

Decision Variables:

$A_{t}=\left\{\begin{array}{l}1, \text { if the transport is available at that time } \\ 0, \text { Otherwise }\end{array}\right.$

$c_{r}=\left\{\begin{array}{l}1, \text { if the compartment needed for replacement or maintenance is available at that time } \\ 0, \text { Otherwise }\end{array}\right.$

$U_{m}=\left\{\begin{array}{l}1, \text { if the unexpected ad hock maintenance happened } \\ 0, \text { Otherwise }\end{array}\right.$

$U_{f}=\left\{\begin{array}{l}1, \text { if the unexpected failure or shut down happened } \\ 0, \text { Otherwise }\end{array}\right.$

Equation 2 shows the calculation for the time taken for each maintenance activity and equation 3 is the distance from the current stage to the next stage. For the distance, the time taken can be calculated as shown in equation 4.

$t_{m_{n k}}=t_{\text {finish }}-t_{\text {start }}$

$d=\left(d_{\text {stage } n+1}-d_{\text {stage } n}\right)$

$t_{d}=d /$ speed

If there is more than one machine needs to be inspected at the stage n, equation 5 will be used where $a$ refers to the machines or inspection that need to be done.

$t_{N}=\sum_{a=1}^{b}\left[N_{c a}\left(t_{m a}\right)\right]$ 
Then, for the uncertainty, we will choose the maximum value of all the uncertainty as it shows the highest priority to be maintained first during stage $m_{n}$ at station $\mathrm{k}$ in equation 6 .

$\max \left[A_{t}+c_{r}+U_{m}+U_{f}\right]^{m_{n k}}$

Then, time will be measured for each maintenance process at stage $n$ and state $m_{n k}$.

$T_{n k}=\min \left[t_{d}+t_{m_{n k}}+t_{N}\right]$

Therefore,

$x_{n}=\max \left[A_{t}+c_{r}+U_{m}+U_{f}\right]^{m_{n k}} \cap \min \left[t_{D}+t_{m_{n k}}+t_{N}\right]$

In this part, the calculation will be based on this functional equation and the decision can be made from the calculation where $x_{n}$ is the combination of the minimisation of time and the uncertainty.

Contribution Function $\left(D_{n}\left(m_{n k}, x_{n k}\right)\right)$ : This function is providing the value at stage $n$ given the decision variable $x_{k n}$. According to the Dynamic Programming Principle, the calculation is done to get the value of each state for decision making processed.

$D_{n}\left(m_{n k}, x_{n k}\right)=T_{n k}+\min \sum_{i=1}^{k} T_{n i}$

$D_{n}^{*}\left(m_{n k}\right)=\min _{x_{n k=0,1, \ldots, m_{n k}}} D_{n}\left(m_{n k}, x_{n k}\right)$

Therefore,

$D_{n}\left(m_{n k}, x_{n k}\right)=T_{n k}+D_{n+1}^{*}\left(m_{n k}-x_{n k}\right)$

Recursive equation $\left(D_{n}^{*}\left(m_{n k}\right)\right)$ : For the recursive relationship,

$D_{n}^{*}\left(m_{n k}\right)=\min _{x_{n k}=0,1, \ldots, m_{n k}}\left\{t_{N}+D_{n+1}^{*}\left(m_{n k}-x_{n k}\right)\right\}$

For $n=1,2, \ldots, n, n+1$.

For dynamic programming, there are two types of recurrent process: forward formulation and backwards formulation. The forward formulation is the type of formulation that starts with the initial stage which is stage 1, and the input will be carrying forward to find the next stage solution until the whole solution can be solved. For backwards formulation, the solution starts with the final stage, and the dynamic programming determines the optimal value for each stage and continues backwards until the solution for stage 1 is obtained. In this research, the forward formulation will be used as the maintenance schedule start with the first stage and from the calculation of each stage, the decision can be made.

Then, the matrix $\left[a_{i j}\right]$ that represent the topology graph in Figure 5 will be introduced. The matrix $\left[a_{i j}\right]$ can only get from the topology graph structure that is developed based on the real life situation. Figure 6 shows the matrix the for topology graph. Based on the matrix, if $a_{i j}=1$, it denotes that the node $i$ can directly get to the node $j$ meanwhile, if $a_{i j}=0$, the node $i$ cannot directly get to node $j$. By relating this matrix with Figure 5, the arrows show the relationship between $i$ and $j$ in the matrix. This matrix will help to get a better understanding of the network and help to get the convenient calculation. 


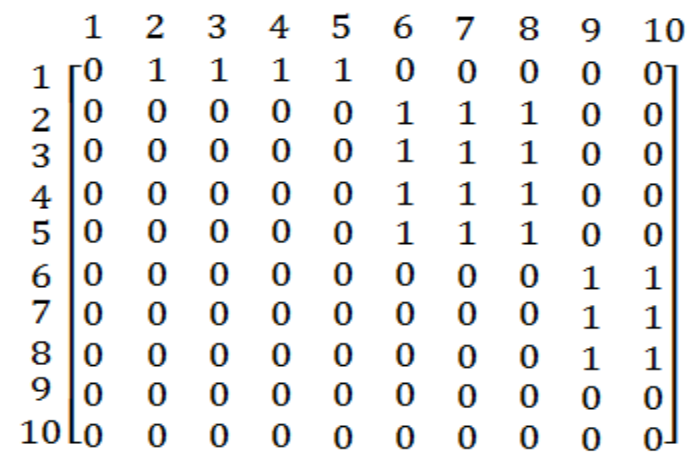

Figure 6: Matrix

\section{DISCUSSION}

Breakdown gives a high impact to the operations, no matter in the production line or utility providers. In the utility providers such as electricity and water provider or sewage treatment plants, breakdown can disturb the operation and will disrupt our daily lives. Maintenance needs to perform based on its schedule to the equipment or facilities to avoid any breakdown or to minimise the breakdown. Uncertainties that exist in the process will prevent the organisation from executing the schedule smoothly. These uncertainties differ from one organisation to another as each of the organisations has a different type of machines and equipment and different plant layout. Therefore, each of these uncertainties needs to be managed properly.

\section{CONCLUSION}

The dynamic programming algorithm was proposed to solve the maintenance scheduling problem as it can deal with the uncertainty that happened during the maintenance process. Therefore, to complete this research, this algorithm will be applied to the industry or company that required a schedule for their maintenance process then the data from that industry or company will be computed to produce new schedule. By considering the uncertainty, the schedule generated is a fully optimise schedule which minimising time that needed for the whole maintenance process.

\section{REFERENCES}

[1] Baker, K. R. (1974). Introduction to sequencing and scheduling: John Wiley \& Sons.

[2] Chen, J., Lee, F. N., Breipohl, A. M., \& Adapa, R. (1995). Scheduling direct load control to minimize system operational cost. IEEE Transactions on Power Systems, 10(4), 1994-2001. http://doi.org/10.1109/59.476068

[3] Nakajima, S. and Shirase, K. 1992. New TPM development program for assemby process (in Japanese). JIPM Solution, Tokyo, Japan

[4] Zandieh, M., Khatami, A. R., \& Rahmati, S. H. A. (2017). Flexible job shop scheduling under condition-based maintenance: Improved version of imperialist competitive algorithm. Applied Soft Computing, 58, 449-464. https://doi.org/10.1016/j.asoc.2017.04.060

[5] M.A. Mansor, A. O. and S. S. (2012). Knowledge Management for Maintenance Activities in the Manufacturing Sector. International Journal of Automotive and Mechanical Engineering (IJAME), 5 (June), 612-621. 
[6] Zandieh, M., Khatami, A. R., \& Rahmati, S. H. A. (2017). Flexible job shop scheduling under condition-based maintenance: Improved version of imperialist competitive algorithm. Applied Soft Computing, 58, 449-464. http://doi.org/10.1016/j.asoc.2017.04.060

[7] Froger, A., Gendreau, M., Mendoza, J. E., Pinson, É., \& Rousseau, L.-M. (2015). Maintenance scheduling in the electricity industry: A literature review. European Journal of Operational Research, 251(3), 695-706. http://doi.org/10.1016/j.ejor.2015.08.045

[8] Ye, X., Xia, X., Zhang, L., \& Zhu, B. (2015). Optimal maintenance planning for sustainable energy efficiency lighting retrofit projects by a control system approach. Control Engineering Practice, 37, 1-10. http://doi.org/10.1016/j.conengprac.2014.12.014

[9] Azhar, N. A. C., \& Mansor, M. A. (2013). Development of Plant Maintenance Management System(PMMS): A Case Study. International Conference on Mechanical Engineering Research (ICMER2013), 1-3 July 2013, (July), 1-3.

[10] Chen, W.-J., \& Liao, C.-J. (2005). Scheduling with different maintenance policies in a textile company. Journal of Quality in Maintenance Engineering, 11(1), 43-52. http://doi.org/10.1108/13552510510589361.

[11] Kocatepe, O. (2014). An application framework for scheduling optimization problems. 2014 IEEE 8th International Conference on Application of Information and Communication Technologies (AICT), 1-4. http://doi.org/10.1109/ICAICT.2014.7035950

[12] Reklaitis, G. V. (2000). Overview of Planning and Scheduling Technologies. Latin American Aplied Research, 293(30), 285-293.

[13] Aguilar, R. J. (1973). Systems analysis and design in engineering, architecture, construction, and planning: Prentice-Hall.

[14] Lieberman, G. J., \& Hillier, F. S. (2010). Introduction to OPERATIONS RESEARCH Ninth Edition (Ninth Edit). Mc Graw Hill. 